\title{
Revisión estratigráfica de Punta Coyote (Baja California Sur, México) e implicaciones para el volcanismo de la Sierra Madre Occidental y el arco Comondú
}

\author{
Stratigraphic review of Punta Coyote (Baja California Sur, Mexico) \\ and implications for the volcanism of the Sierra Madre Occidental \\ and the Comondu arc
}

\author{
M.J. Puy Alquiza ${ }^{1}$, R. Miranda Avilés ${ }^{1}$, M. López Martínez²
}

\begin{abstract}
RESUMEN
Se presentan nuevos datos sobre la estratigrafía y sedimentología de la sucesión volcanosedimentaria de la Unidad Inferior del Grupo Comondú (UIGC) al noreste de la ciudad de La Paz, en Punta Coyote, Baja California Sur, México. El análisis de facies, así como las nuevas dataciones ${ }^{40} \mathrm{Ar} /{ }^{39} \mathrm{Ar}$ permitieron interpretar la evolución del ambiente sedimentario y los pulsos volcánicos de la UIGC en Punta Coyote. De acuerdo al análisis realizado, el área estudiada formó parte de la cuenca de arco delantero al oeste del arco volcánico de la Sierra Madre Occidental durante el Oligoceno inferior al Mioceno inferior. El ambiente sedimentario de la unidad inferior del Grupo Comondú evolucionó inicialmente como un ambiente fluvial trenzado progradando a un ambiente de abanico aluvial donde llegaron a depositarse tres unidades tobáceas: toba Los Azabaches $(30.6 \pm 0.4 \mathrm{Ma})$, toba La Capilla y toba El Oro (22.0 \pm $0.4 \mathrm{Ma}$ ). La sucesión estudiada aporta nueva información en relación a la evolución estratigráfica del arco volcánico Oligoceno-Mioceno en la Península de Baja California y su correlación con los depósitos del Supergrupo Volcánico Superior de la Sierra Madre Occidental al noroeste de México.
\end{abstract}

Palabras clave: Arco volcánico, Sierra Madre Occidental, Península de Baja California, Grupo Comondú, Punta Coyote.

\begin{abstract}
We present new stratigraphic evidence for a migration of the Upper Volcanic Supergroup that formed the Sierra Madre Occidental (SMO) volcanic arc in northwestern Mexico during the Oligocene-Miocene. Particularly we discuss the transition from the frontal arc to the Comondú volcanic arc in Punta Coyote, Baja California Peninsula. This information complements the knowledge of the stratigraphic evolution of the Oligocene-Miocene volcanic arc in Baja California and its correlation with the Upper Volcanic Supergroup of the Sierra Madre Occidental in northwestern Mexico. We present new stratigraphic and sedimentologic data of the volcanosedimentary succession of the lower unit of the Comondú group near of La Paz, Baja California Sur, Mexico, in Punta Coyote. Facies analysis and new ${ }^{40} \mathrm{Ar} /{ }^{39} \mathrm{Ar}$ dating allowed to interpret the sedimentary environment and evolution of volcanic pulses in the lower unit of the Comondú group in Punta Coyote. The study area was part of the forearc basin westward to the SMO volcanic arc during the Oligocene to early Miocene. The sedimentary environment of the lower unit of the Comondú group was initially a braided river system that prograded to an alluvial fan environment and three tuffs units: Los Azabaches tuff $(30.6 \pm 0.4 \mathrm{Ma})$, La Capilla and El Oro tuffs $(22.0 \pm 0.4 \mathrm{Ma})$. The succession studied provides new stratigraphic information related to the evolution of the Oligocene-Miocene volcanic arc in the Baja California Peninsula and its correlation with the Upper Volcanic Supergroup of the SMO in northwestern Mexico.
\end{abstract}

Key words: Volcanic arc, Sierra Madre Occidental, Baja California Peninsula, Comondú Group, Punta Coyote.

\footnotetext{
1 Departamento de Minas, Metalurgia y Geología, Universidad de Guanajuato Ex-Hacienda de San Matías S/N Colonia San Javier, C.P. 36020, Guanajuato, Gto. México. Fax: 01 (473) 73238 64. Email: yosune@ quijote.ugto.mx

2 Departamento de Geología, División de Ciencias de la Tierra, CICESE, Ensenada, Baja California, México.
} 


\section{Introducción}

Gran parte del Paleógeno y parte del Neógeno el margen oeste de Norteamérica actuó como un límite de placa convergente, en donde la placa Farallón era subducida bajo la placa Norteamericana (Atwater, 1970; Gastil et al., 1981). El arco volcánico establecido por este proceso de subducción, formó la Sierra Madre Occidental (SMO) cuya parte más reciente constituye la provincia ígnea silícea más grande de Norteamérica (McDowell \& Keizer, 1977; McDowell \& Clabaugh, 1979). La SMO está constituida por cinco conjuntos ígneos (Ferrari et al., 2007): 1) rocas plutónicas y volcánicas del Cretácico superior-Paleoceno; 2) rocas volcánicas andesíticas y en menor medida, dacítico-riolíticas del Eoceno, tradicionalmente agrupadas en el denominado Complejo Volcánico Inferior (CVI) (McDowell \& Keitzer, 1977); 3) ignimbritas silícicas emplazadas en su mayoría en dos pulsos, en el Oligoceno temprano (32-28 Ma), el Mioceno temprano (24-20 Ma), denominadas Supergrupo Volcánico Superior (SVS); 4) coladas basáltico-andesíticas transicionales extravasadas después de cada pulso ignimbrítico, y 5) volcanismo post-subducción constituido por coladas de basaltos alcalinos e ignimbritas emplazados en diferentes episodios del Mioceno tardío, Plioceno y Cuaternario y que se relacionan con la separación de la Península de Baja California del resto del continente.

El Supergrupo Volcánico Superior (SVS) está constituido por una sucesión ignimbrítica con espesores de más de 1000 metros que conforman gran parte del volumen de la SMO (McDowell \& Clabaugh, 1979). Ferrari et al. (2002), determinaron un patrón general para la ocurrencia de los pulsos ignimbríticos de la SMO con base en la distribución de edades de las rocas. De acuerdo a estos autores, el arco generado entre 32 y 28 Ma se distribuye en una franja NNW, mientras que las edades disponibles en el Mioceno inferior permiten dividir el volcanismo silícico en dos pulsos, uno a los 23.5 Ma y otro a $21 \mathrm{Ma}$ (fig. 1).

En la Península de Baja California, el SVS está representado por el Grupo Comondú (GC) que tiene un rango de edad entre 30 y $12 \mathrm{Ma}$ en la porción central (Gastil et al., 1979; McFall, 1968; Mclean, 1988; Umhoefer et al., 2001), mientras que hacia el sur de la península se han reportado edades más jóvenes de 25 Ma (Hausback, 1984; Sawlan \& Smith, 1984; Sawlan, 1991). Sin embargo, en el presente trabajo se reportan nuevas edades, algunas de éstas más antiguas que las previamente reportadas para la porción sur, correlacionables con cada uno de los dos pulsos magmáticos del SVS reportados por Ferrari et al. (2002) en Nayarit.

Las rocas volcánicas y volcanoclásticas que conforman la Sierra Madre Occidental fueron depositadas cuando la actual Península de Baja California formaba parte de la placa Norteamericana (Atwater, 1970; Stock \& Hodges, 1989) (fig. 1). Durante el Mioceno y Plioceno la Península de Baja California fue separada de la placa Norteamérica, conformando el rift actual del Golfo de California (Atwater, 1970; Stock \& Hodges, 1989; Martín Barajas et al., 2000; Miranda Avilés et al., 2005).

Uno de los problemas para el estudio de la estratigrafía de la SMO es la dificultad de acceso, la escasez de afloramientos de las unidades pre-Oligoceno y el intenso fallamiento extensional postEoceno (Ferrari et al., 2007). Sin embargo, en Baja California Sur existen excelentes afloramientos de las rocas volcánicas y volcaniclásticas relacionadas al Supergrupo Volcánico Superior (SVS) o Grupo Comondú (30-12 Ma).

El objetivo de este artículo es presentar nuevos datos sobre la estratigrafía y sedimentología de la sucesión volcanosedimentaria del Grupo Comondú que es correlacionable al Supergrupo Volcánico Superior (SVS) de la Sierra Madre Occidental. Particularmente se analizan los pulsos volcánicos del Grupo Comondú en Punta Coyote, al sur de la Península de Baja California (fig. 1). Esta nueva información complementa el conocimiento sobre la evolución estratigráfica del arco volcánico Oligoceno-Mioceno en la Península de Baja California y su correlación con las rocas volcánicas del SVS de la Sierra Madre Occidental al noroeste de México.

El estudio se enfocó en los afloramientos de rocas volcánicas intercaladas por areniscas rojas y conglomerados, que estratigráficamente corresponden a la unidad inferior del Grupo Comondú (UIGC) al noreste de la ciudad de La Paz, en Punta Coyote (fig. 2). Estas rocas fueron descritas en el área de estudio como rocas pre-volcanismo (Aranda Gómez y Pérez Venzor, 1988) o miembro salinas (Hausback, 1984).

\section{Metodología}

Con el fin de conocer el ambiente de sedimentación del UIGC se levantaron ocho secciones estratigráficas en el área de Punta Coyote: sección Punta 


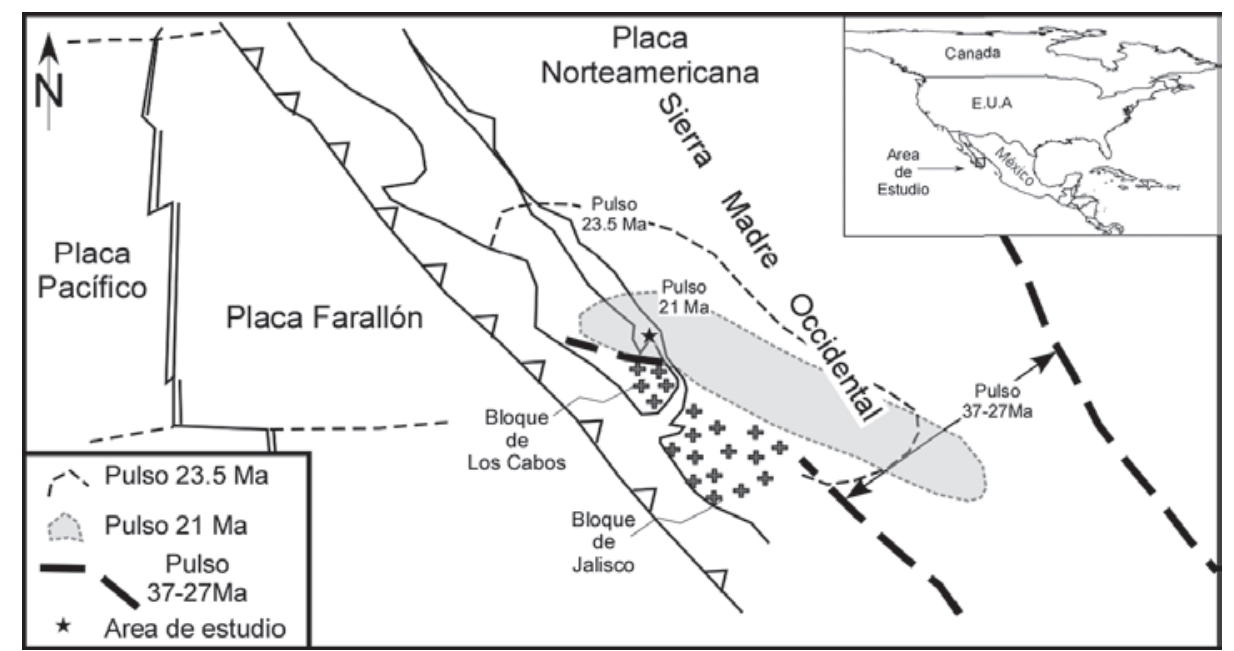

Fig. 1.-Localización del área de estudio. Esquema donde se muestran el marco tectónico generalizado del oeste de México y los pulsos volcánicos entre 37 y $21 \mathrm{Ma}$ (modificado de Ferrari et al., 2002).

Arranca Cabellos; sección Arroyo Cachimba, sección Cañada Los Hornos, sección Cañada La Pedrera, sección Cañada Portezuelo-Pichilingue, sección Arroyo Portezuelo-Pichilingue, sección Arroyo El Oro y sección Arroyo Santa Victoria (figs. 2 y 3). La interpretación de la historia sedimentológica del área de estudio se realizó con base en el análisis de facies que consistió en definir litofacies utilizando la nomenclatura de facies fluvial propuesta por Miall (1978). En cuanto a la división de litofacies de las rocas volcánicas del UIGC, se realizó tomando en cuenta los trabajos acerca de las sucesiones volcánicas de Cas \& Wright (1982). La interpretación se llevó a cabo utilizando la clasificación propuesta por Branney \& Kokelaar (2003). En este trabajo se fecharon dos muestras de roca volcánica por el método ${ }^{40} \mathrm{Ar} /{ }^{\beta 9} \mathrm{Ar}$. Las mediciones se efectuaron en concentrados de feldespato, los cuales fueron preparados siguiendo la metodología del Laboratorio de Geocronología del CICESE (Cerca Martínez et al., 2000).

Las muestras fueron irradiadas en el reactor nuclear de la Universidad de McMaster en Canadá. El análisis isotópico de argón se realizó en el Laboratorio de Geocronología del CICESE con el espectrómetro de masas VG5400 utilizando el láser de iones de argón, marca Coherent Innova 70 como sistema de calentamiento. Para la muestra RM0105 se preparó un concentrado de plagioclasa y de feldespato de potasio para la muestra RM0205. Como monitores de irradiación se utilizó el sanidino TCR-2 de $27.87 \pm 0.04 \mathrm{Ma}$, las muestras de los monitores de irradiación se distribuyeron alrededor de las muestras. Los isótopos de argón se corrigieron por blanco, decaimiento de ${ }^{37} \mathrm{Ar}$ y ${ }^{39} \mathrm{Ar}$, discriminación y reacciones de interferencia de $\mathrm{Ca}, \mathrm{K}$ y $\mathrm{Cl}$. En todos los cálculos se utilizaron las constantes recomendadas por Steiger \& Jager, 1997 y las ecuaciones de York et al., 2004 se utilizaron en todas las rutinas de cálculo de líneas rectas. En la plagioclasa RM0105 se realizaron dos experimentos de fusión en un paso y un experimento de calentamiento en etapas en el cual se colectaron únicamente dos fracciones. Para el feldespato de potasio RM0205 se realizó un experimento de fusión en un paso y cuatro experimentos de calentamiento en etapas. Una síntesis de los resultados de ${ }^{40} \mathrm{Ar}-{ }^{39} \mathrm{Ar}$ se presenta en la tabla 2 y en la figura 4. En la figura 4 se presenta para las muestras RM0105 y RM0205, espectro de edad (a y d), diagrama de ${ }^{37} \mathrm{Ar}_{\mathrm{Ca}}{ }^{39} \mathrm{Ar}_{\mathrm{K}}$ (b y e) y diagrama de correlación ${ }^{36} \mathrm{Ar} /{ }^{40} \mathrm{Ar}$ versus ${ }^{39} \mathrm{Ar} /{ }^{40} \mathrm{Ar}$ (c y f). El bajo contenido de potasio de la muestra RM0105 no permitió realizar experimentos de calentamiento en etapas detallados. Los datos obtenidos son consistentes tanto en el espectro de edad como en el diagrama de correlación rindiendo edades estadísticamente indistinguibles. En el diagrama ${ }^{37} \mathrm{Ar}_{\mathrm{Ca}}{ }^{\beta 9} \mathrm{Ar}_{\mathrm{K}}$ puede observarse que la composición de $\mathrm{Ca} / \mathrm{K}$ de esta muestra es homogénea (fig. 4b). La mejor estimación de la edad de la toba El Oro se toma del diagrama de correlación (fig. 4c) en donde los cuatro datos claramente se alinean $(\mathrm{MSWD}=0.8)$ para rendir una edad de isócrona de $22.0 \pm 0.4 \mathrm{Ma}$ con un $\left({ }^{40} \mathrm{Ar} /{ }^{36} \mathrm{Ar}\right)_{\mathrm{i}}$ de $302 \pm 10$. 


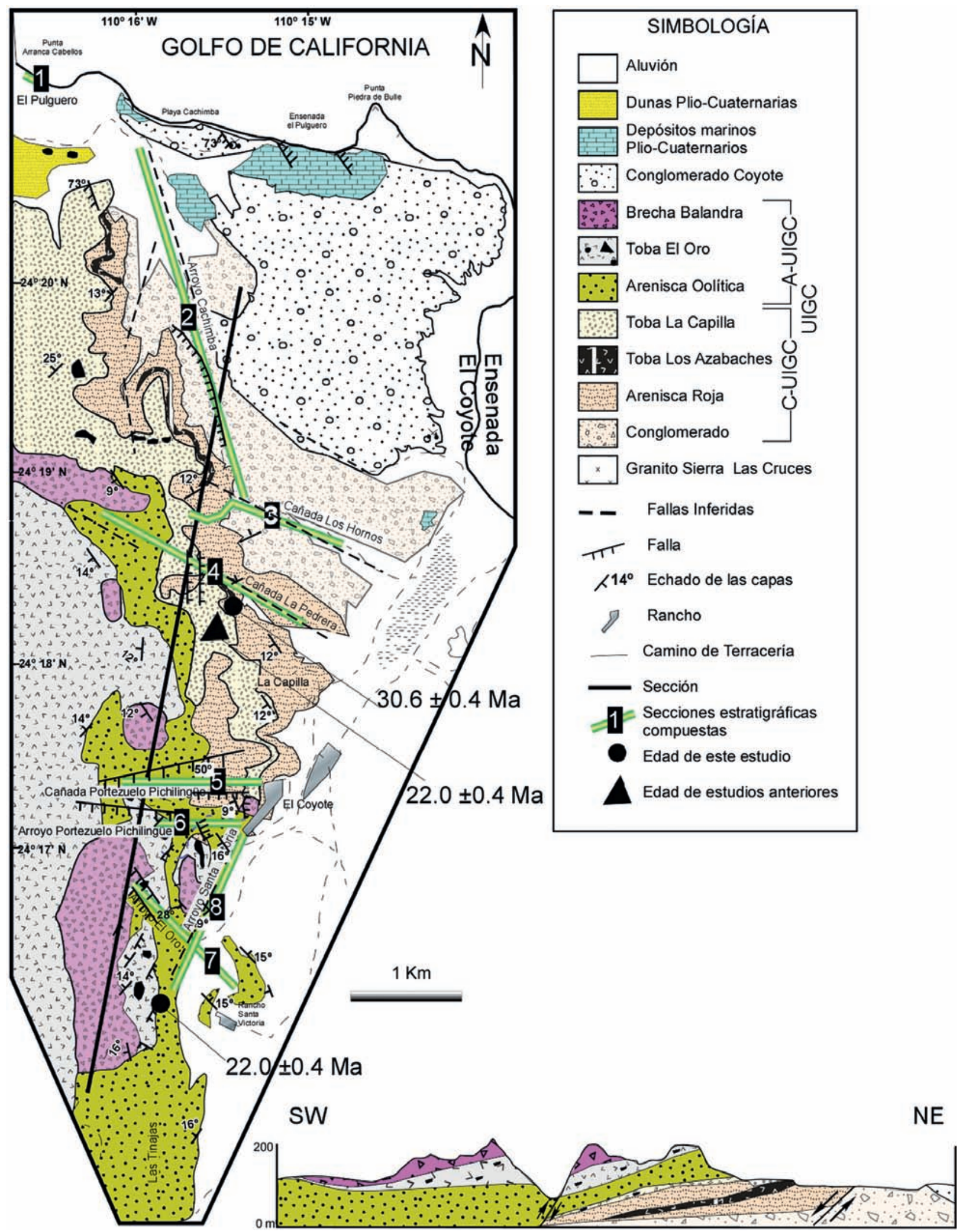

Fig. 2.-Carta geológica del área de estudio. 


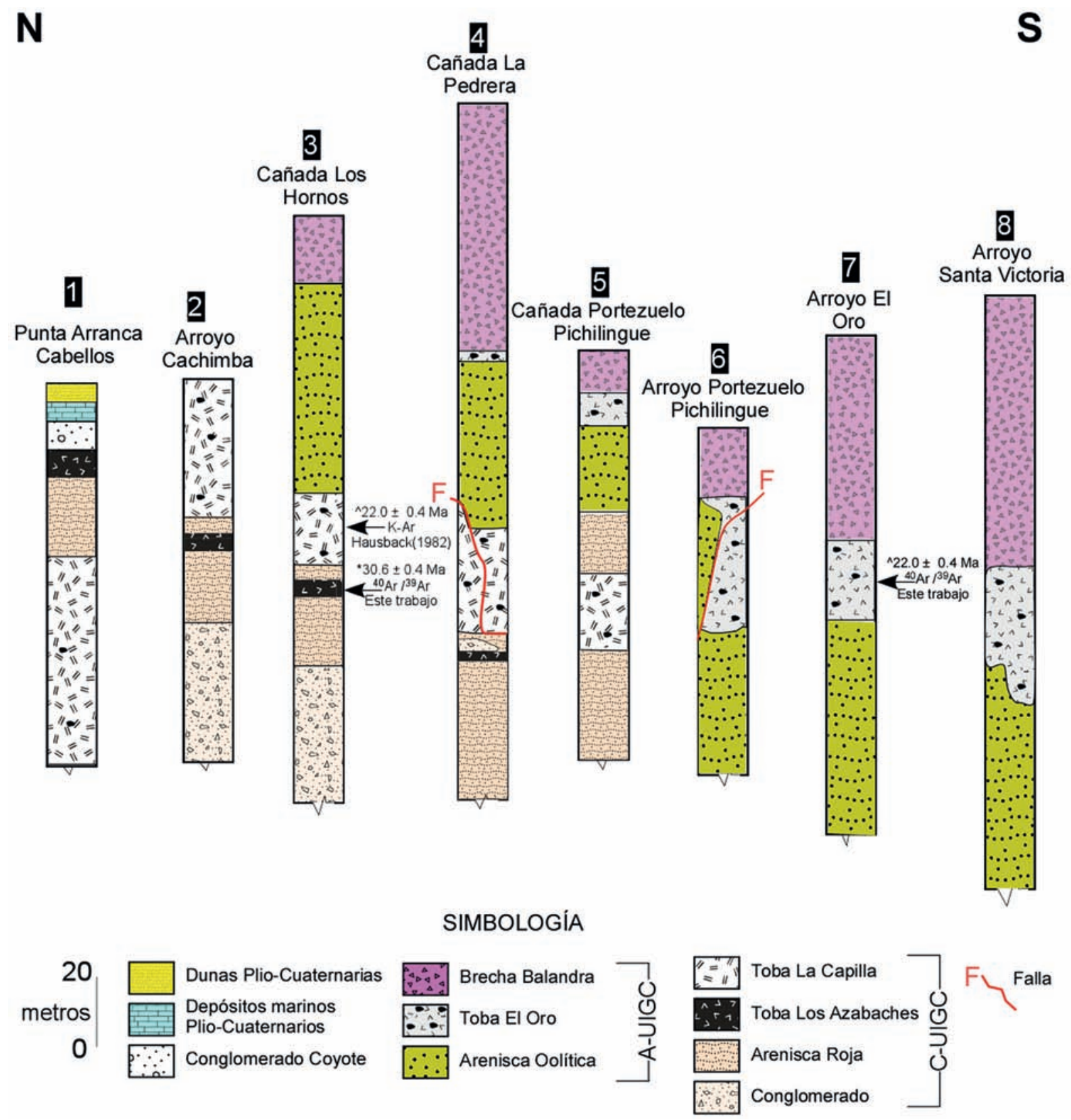

Fig. 3.-Columnas estratigráficas medidas en el área estudiada.

El feldespato de potasio RM0205, se analizó en mayor detalle. En el espectro de edad (fig. 4d) puede observarse que se obtuvieron edades consistentes para todos los experimentos realizados. En el diagrama ${ }^{37} \mathrm{Ar}_{\mathrm{Ca}}{ }^{39} \mathrm{Ar}_{\mathrm{K}}$ (fig. 4e) puede observarse que la muestra tiene una composición homogénea, únicamente dos fracciones, liberadas a baja temperatura, rinden valores de ${ }^{37} \mathrm{Ar}_{\mathrm{Ca}}{ }^{39} \mathrm{Ar}_{\mathrm{K}}>$ que 1 ; sin embargo, las fracciones colectadas aplicando mayor potencia con el láser, definen una meseta. Además, las edades de meseta obtenidas son estadísticamente indistinguibles $(30.8 \pm 0.3$ y $30.7 \pm 0.2 \mathrm{Ma})$. Con excepción de las fracciones liberadas a baja temperatura, los datos obtenidos en todos los experimentos se combinaron en el diagrama de correlación (fig. 4f) para definir una edad de isócrona de $30.6 \pm 0.4 \mathrm{Ma}$, la cual se tomó como la mejor estimación de la edad de la toba Los Azabaches. 
a)

b)

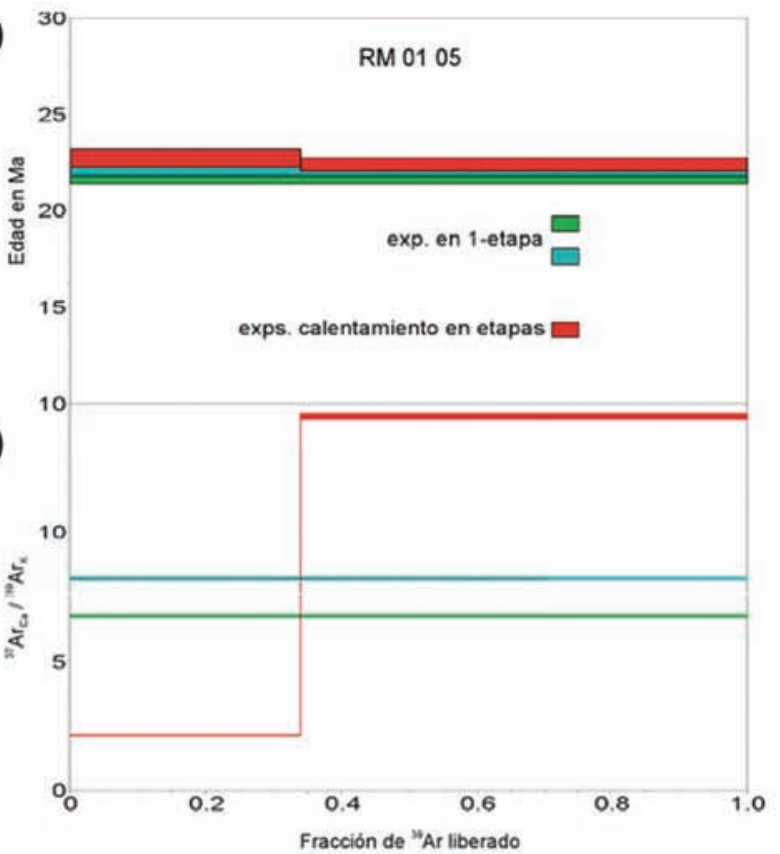

c)

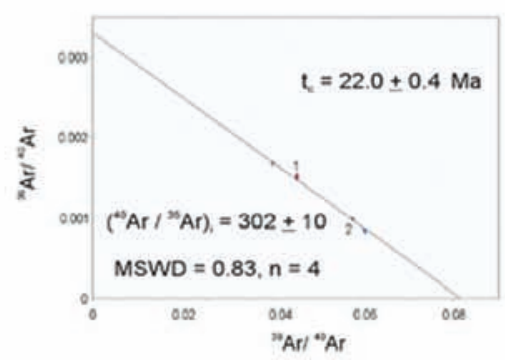

d)

e)

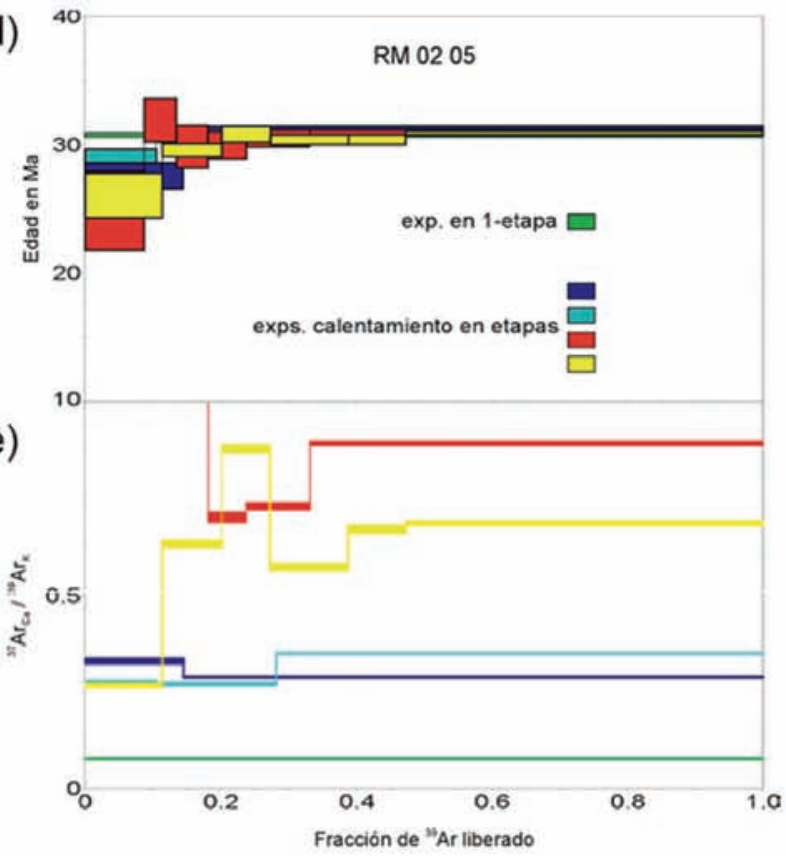

f)

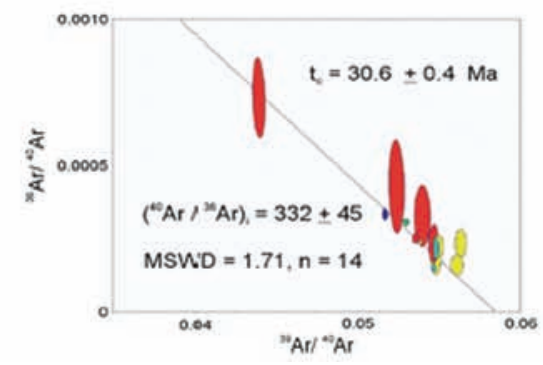

Fig. 4.-Diagramas de seudoespectros de edad y correlación ${ }^{36} \mathrm{Ar} /{ }^{40} \mathrm{Ar} v \mathrm{~S}^{39} \mathrm{Ar} /{ }^{40} \mathrm{Ar}$ de las muestras RM0105 y RM0205.

\section{Antecedentes de la unidad inferior del Grupo Comondú (UIGC)}

En la Península de Baja California se han descrito y localizado rocas que pertenecen a la unidad inferior del Grupo Comondú (UIGC) en Bahía Concepción, Loreto, San Juan de la Costa, Punta San Telmo, Punta Coyote, Tembabiche, en los alrededores de la ciudad de La Paz y en El Pulguero, (McFall, 1968; Gastil et al., 1979; Hausback, 1984; Aranda Gómez \& Pérez Venzor, 1988; McLean, 1988; Schwennicke, 2000; Umhoefer et al., 2001). En Bahía Concepción, McFall (1968) denominó Formación El Salto a un grupo de rocas sedimentarias conformadas por areniscas rojas con estratificación cruzada y rocas volcánicas fechadas por K/Ar en biotita $(28.1 \pm 0.9 \mathrm{Ma})$, consideradas como la parte más vieja del Grupo Comondú. Schwennicke (2000), describió depósitos de areniscas rojas inter- caladas con conglomerados, fanglomerados y tobas en Tembabiche. En Loreto, Umhoefer et al. (2001) dividió al Grupo Comondú en tres unidades, la unidad clástica inferior dominada por areniscas rojas con estratificación cruzada, conglomerados y tobas con relaciones de facies complejas; la unidad media constituida por brechas y flujos de lava de $17.8 \pm 0.7 \mathrm{Ma}$ fechados por K/Ar en roca total (Sawlan \& Smith, 1984) y la unidad superior compuesta por flujo de lava y brecha fechados por Umhoefer et al. (2001), con $14.6 \pm 0.09 \mathrm{Ma}\left({ }^{40} \mathrm{Ar} /{ }^{39} \mathrm{Ar}\right.$ en hornblenda).

En el área del presente estudio las rocas de la UIGC, fueron nombradas miembro Salinas por Hausback (1984) y descritas como la parte basal del Grupo Comondú. El miembro Salinas fue definido como una secuencia de areniscas, conglomerados volcánicos, tobas de flujos de cenizas riolíticas, lahares andesíticos y flujos de lava con una edad $\mathrm{K} / \mathrm{Ar}$ de $25 \pm 0.6 \mathrm{Ma}$ a $23.2 \pm 1.6 \mathrm{Ma}$. Posterior- 
mente Aranda Gómez y Pérez Venzor (1988) realizaron un estudio geológico en Punta Coyote, donde dividieron las rocas de la zona en rocas pre-volcanismo, secuencia volcano-sedimentaria y roca postvolcanismo. Las rocas pre-volcanismo corresponden a la UIGC del presente trabajo, la secuencia volcano-sedimentaria y roca post-volcanismo corresponden a la unidad media y unidad superior del Grupo Comondú sensu Umhoefer et al. (2001).

Al NE del área estudiada, los depósitos de la UIGC se encuentran cubiertos por conglomerados cuaternarios del Conglomerado Coyote (Aranda Gómez \& Pérez Venzor, 1988) y por depósitos de dunas y terrazas marinas Plio-cuaternarias.

\section{Estratigrafía}

El término Formación Comondú fue usado primero por Heim (1922) para areniscas y conglomerados expuestos cerca del poblado de Comondú, $45 \mathrm{~km}$ al oeste de Loreto. Posteriormente, Beal (1948) usa el nombre de Formación Comondú para designar a las rocas volcánicas del Cenozoico medio que afloran en la parte media y sur de la Península de Baja California. McFall (1968) realizó la cartografía del área de Bahía Concepción e introduce el nombre de Grupo Comondú. Por último (Umhoefer et al., 2001 y Demant, 1975) proponen que las rocas designadas Formación Comondú sean llamadas Grupo Comondú. En el presente trabajo no proponemos nuevas formaciones para el Grupo Comondú, sin embargo, las unidades que definimos son candidatas a ser designadas formaciones formales con estudios más detallados orientados a cumplir todos los criterios del Código de Nomenclatura Estratigráfica para Norteamérica (NACSN, 1983, 2005). Las unidades que se describen a continuación son correlacionables con la Formación El Salto descrita en Bahía Concepción por McFall (1968), con las rocas pre-volcanismo descritas por Aranda Gómez \& Pérez Venzor (1988), con las unidades informales en las que Umhoefer et al. (2001) divide al Grupo Comondú cerca de Loreto y con rocas volcánicas genéticamente relacionadas a los pulsos volcánicos del SVS de la SMO descritos por Ferrari et al. (2002).

\section{Análisis de facies}

En el área estudiada, los depósitos de la UIGC tienen un espesor máximo de $300 \mathrm{~m}$. En su base se encuentran constituidos por una secuencia de conglomerados, areniscas rojas y toba, mientras que la parte superior la conforman depósitos de areniscas oolíticas, conglomerados, depósitos de flujos de escombros, tobas y brechas volcánicas (figs. 5 y 6). Las facies y asociaciones de facies de la UIGC fueron descritas tomando en cuenta las diferencias en el tamaño de grano, textura, estructuras primarias, composición y geometría de las capas. Para facilitar su descripción fueron divididas en: C-UIGC (depósitos de la base de la unidad inferior Comondú) y A-UIGC (depósitos de la parte superior de la unidad inferior Comondú).

\section{Depósitos de la base de la unidad inferior Comondú (C-UIGC)}

Se caracteriza por una secuencia de conglomerados y areniscas rojas con estratificación cruzada de $160 \mathrm{~m}$ de espesor (tabla 1) y se distribuyen al norte y centro en el área estudiada (Cañada Los Hornos y Cañada La Pedrera). Esta secuencia representa los depósitos más antiguos dentro del Grupo Comondú.

Tabla 1.-Litofacies de la unidad inferior Comondú (UIGC)

\begin{tabular}{|c|c|c|c|c|c|}
\hline \multirow[b]{2}{*}{ Facies } & \multirow[b]{2}{*}{ Estructuras sedimentarias } & \multirow[b]{2}{*}{ Tamaño grano } & \multicolumn{2}{|c|}{ Tamaño de capa } & \multirow[b]{2}{*}{ Interpretación } \\
\hline & & & Espesor & Longitud & \\
\hline Gms & Ninguna & Grava & $1 \mathrm{~m}$ & $1 \mathrm{~m}$ & Depósitos de flujos de gravedad \\
\hline $\mathrm{Gm}$ & Imbrincación & Grava & 0.5 a $8 \mathrm{~m}$ & Decenas de metros & Canales menores \\
\hline Gh & Estratificación horizontal & Grava & 0.5 a $8 \mathrm{~m}$ & Decenas de metros & Bedforms lingoidales \\
\hline $\mathrm{Sm}$ & Gradación inversa & Arena media & $1 \mathrm{~m}$ & $1 \mathrm{~ms}$ & Depósitos de flujos de gravedad \\
\hline St & Estratificación cruzada & Arena gruesa a media & 1 a $10 \mathrm{~m}$ & 1 a $3 \mathrm{~m}$ & Dunas, regímenes de flujos bajos \\
\hline $\mathrm{Sh}$ & Estratificación horizontal & Arena gruesa a media & 0.3 a $0.7 \mathrm{~m}$ & 1 a $2 \mathrm{~m}$ & Canales menores \\
\hline Ss & Ninguna & Arena gruesa a media & 0.3 a $0.7 \mathrm{~m}$ & 1 a $2 \mathrm{~m}$ & Canales someros \\
\hline $\mathrm{Fm}$ & Grietas de desecación & Limo & 0.1 a $0.5 \mathrm{~m}$ & $3 \mathrm{~m}$ & Planicie de inundación \\
\hline Fsm & Masivo & Limo & $0.1 \mathrm{a} 0.6 \mathrm{~m}$ & $3 \mathrm{~m}$ & Planicie de inundación \\
\hline
\end{tabular}



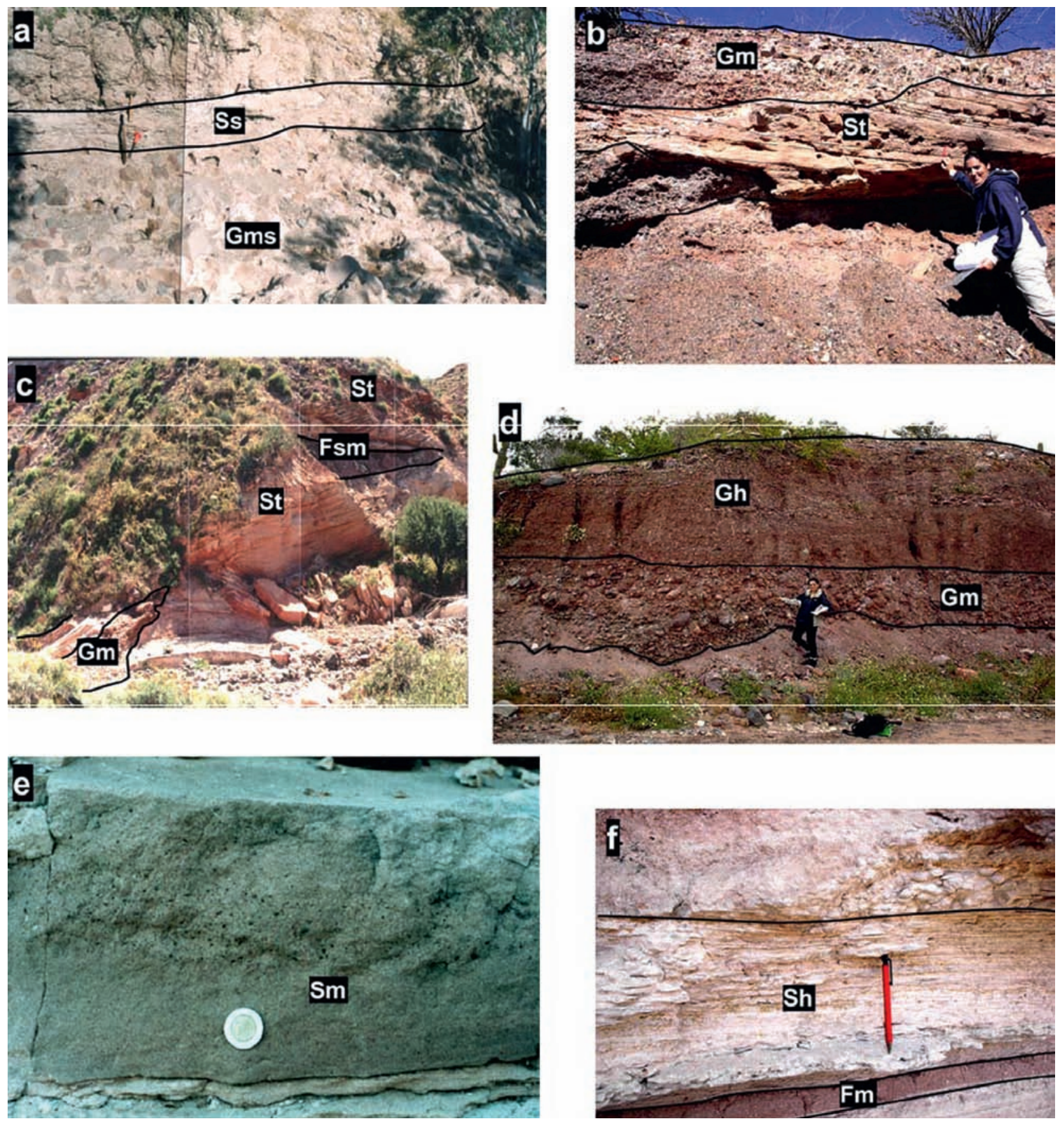

Fig. 5.-Litofacies de la UIGC. a) Facies Gms: Conglomerado matriz soportado, depósitos de flujos de gravedad intercalados con litofacies Ss; b) Facies Gm intercalada con Facies St; c) Facies Gm, St y Fsm; d) Facies Gm: gravas masivas, intercalado con litofacies de gravas con estratificación horizontal; e) Facies Sm: arenisca, gradación inversa; f) Facies Sh: arenisca con laminación horizontal intercalado con litofacies Fm.

Estratigráficamente sobreyace al Granito Sierra de las Cruces (87-94 Ma) (Frizzell et al., 1984) y subyace a las tobas y brechas volcánicas.

Son depósitos de conglomerados masivos, polimícticos con estratificación incipiente a bien estrati- ficados de color rojizo. Los conglomerados se presentan en estratos de 0.5 a $8 \mathrm{~m}$ de espesor con una longitud de decenas de metros y de forma lenticular. Los clastos son del tamaño de las gravas de 3 a $7 \mathrm{~cm}$ de diámetro, de composición volcánica y 

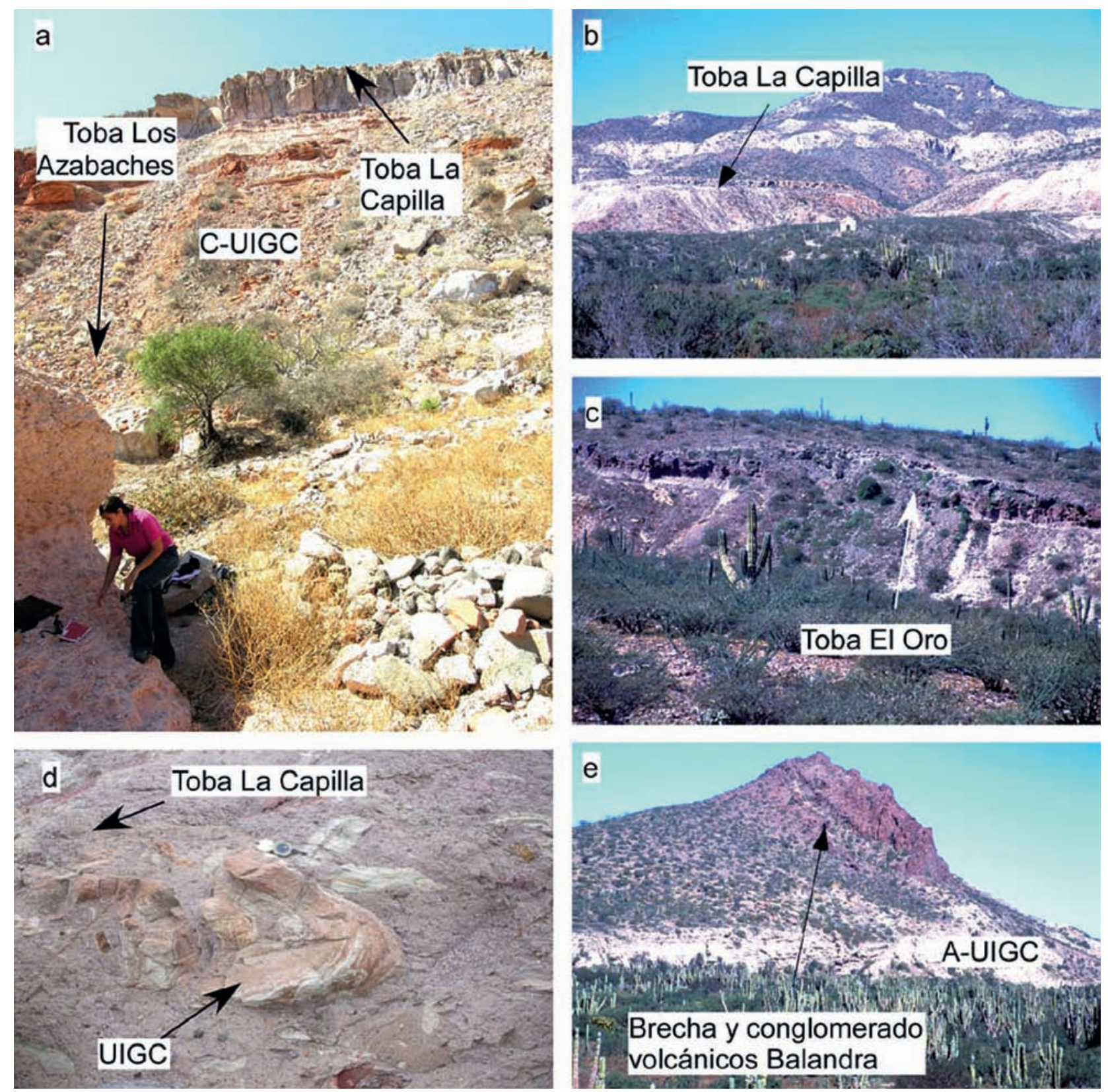

Fig. 6.-Facies volcánicas. a) Toba Los Azabaches; b) Toba La Capilla; c) Toba El Oro; d) Toba La Capilla con bloques accidentados deformados de manera semiplástica (UIGC); e) Brecha y conglomerado volcánicos Balandra.

metamórfica. La forma del clasto es subangular a sub-redondeada de pobre a moderadamente seleccionada. Presentan una geometría cóncava con base erosiva, imbrincación hacia el SW $35^{\circ}$ y SE $40^{\circ}$ y se encuentran intercaladas con facies de conglomerados con estratificación horizontal. Las areniscas son de grano medio a fino de color rojo, de composición subarcósica, ricas en cuarzo y feldespatos. Presentan como estructuras primarias la estratifica- ción cruzada en forma hamacada con echados de $18^{\circ}$ al SE. Se encuentra intercalada con limonitas de $3 \mathrm{~m}$ de longitud y $60 \mathrm{~cm}$ de espesor (tabla 1). Dicha secuencia de areniscas se encuentran intercaladas por la toba Los Azabaches y por una toba que corona a toda la secuencia, denominada toba La Capilla (fig. 7). La primera, toba Los Azabaches, se distribuye en el Arroyo Cachimba y en la Cañada La Pedrera. Corresponde a una toba lítica de $2 \mathrm{~m}$ de 
Tabla 2.—Datos de edad $\mathrm{K} / \mathrm{Ar}\left({ }^{\star}\right)$ y ${ }^{40} \mathrm{Ar} /{ }^{39} \mathrm{Ar}\left({ }^{* \star}\right)$ (ver detalles en tabla 3)

\begin{tabular}{|c|c|c|c|c|c|c|}
\hline $\begin{array}{l}\text { Número } \\
\text { de muestra }\end{array}$ & $\begin{array}{l}\text { Localización } \\
\text { Lat/Long }\end{array}$ & $\begin{array}{l}\text { Tipo } \\
\text { de roca }\end{array}$ & $\begin{array}{l}\text { Mineral } \\
\text { datado }\end{array}$ & Edad (Ma) & $\begin{array}{c}\text { Unidad } \\
\text { Estratigráfica }\end{array}$ & Fuente \\
\hline 317 & $\begin{array}{l}24^{\circ} 18^{\prime} 10^{\prime \prime} \mathrm{N} \\
110^{\circ} 15^{\prime} 41^{\prime \prime} \mathrm{W}\end{array}$ & Toba & Biot & $20.3 \pm 0.5^{*}$ & $\begin{array}{l}\text { Depósitos de la base de la unidad } \\
\text { inferior Comondú (AC-UIGC) }\end{array}$ & 2 \\
\hline RM-01-05 & $\begin{array}{l}24^{\circ} 16^{\prime} 2.4^{\prime \prime} \mathrm{N} \\
110^{\circ} 15^{\prime} 55^{\prime \prime} \mathrm{W}\end{array}$ & $\begin{array}{l}\text { Toba } \\
\text { vítrea }\end{array}$ & Plag & $22.0 \pm 0.4 * *$ & $\begin{array}{l}\text { Depósitos de la parte superior } \\
\text { de la unidad inferior Comondú } \\
\text { (AC-UIGC) }\end{array}$ & 1 \\
\hline Bj4 & $\begin{array}{l}24^{\circ} 17^{\prime} 12^{\prime \prime} \mathrm{N} \\
110^{\circ} 18^{\prime} 20^{\prime \prime} \mathrm{W}\end{array}$ & Toba & Biot & $22.0 \pm 0.4^{*}$ & $\begin{array}{l}\text { Depósitos de la base de la unidad } \\
\text { inferior Comondú (AC-UIGC) }\end{array}$ & 2 \\
\hline 238 & $\begin{array}{l}24^{\circ} 09^{\prime} 05^{\prime \prime} \mathrm{N} \\
110^{\circ} 15^{\prime} 49^{\prime \prime} \mathrm{W}\end{array}$ & Toba & Plag & $23.2 \pm 1.6^{*}$ & $\begin{array}{l}\text { Depósitos de la base de la unidad } \\
\text { inferior Comondú (AC-UIGC) }\end{array}$ & 2 \\
\hline 394 & $\begin{array}{l}24^{\circ} 08^{\prime} 32^{\prime \prime} \mathrm{N} \\
110^{\circ} 16^{\prime} 16^{\prime \prime} \mathrm{W}\end{array}$ & Toba & Biot & $25.0 \pm 0.6^{*}$ & $\begin{array}{l}\text { Depósitos de la base de la unidad } \\
\text { inferior Comondú (AC-UIGC) }\end{array}$ & 2 \\
\hline RM-02-05 & $\begin{array}{l}24^{\circ} 18^{\prime} 30.7^{\prime \prime} \mathrm{N} \\
110^{\circ} 15^{\prime} 48^{\prime \prime} \mathrm{W}\end{array}$ & $\begin{array}{l}\text { Toba } \\
\text { lítica }\end{array}$ & San & $30.6 \pm 0.4^{* *}$ & $\begin{array}{l}\text { Depósitos de la base de la unidad } \\
\text { inferior Comondú (AC-UIGC) }\end{array}$ & 1 \\
\hline
\end{tabular}

Dataciones realizadas por: (1) Este estudio; (2) Hausback (1984); Bio = Biotita, Plag = Plagioclasa, San = Sanidino.

espesor de color rosa salmón con pómez, líticos volcánicos y metamórficos de 1 a $3 \mathrm{~cm}$ de diámetro, contenidos en una matriz afanítica que consiste en intercrecimientos criptocristalino y microcristalino de plagioclasas, cuarzo y biotita. Para la toba Los Azabaches se estimó una edad ${ }^{40} \mathrm{Ar} /{ }^{39} \mathrm{Ar}$ en sanidino. En el espectro de edad (fig. 4d) puede observarse que se obtuvieron edades consistentes para todos los experimentos realizados. En el diagrama ${ }^{37} \mathrm{Ar}_{\mathrm{Ca}}{ }^{\beta 9} \mathrm{Ar}_{\mathrm{K}}$ (fig. 4e) puede observarse que la muestra tiene una composición homogénea, únicamente dos fracciones, liberadas a baja temperatura, rinden valores de ${ }^{37} \mathrm{Ar}_{\mathrm{Ca}}{ }^{\beta 9} \mathrm{Ar}_{\mathrm{K}}>$ que 1 ; sin embargo, las fracciones colectadas aplicando mayor potencia con el láser, definen una meseta. Además, las edades de meseta obtenidas son estadísticamente indistinguibles $(30.8 \pm 0.3$ y $30.7 \pm 0.2 \mathrm{Ma})$ (tabla 3$)$. Con excepción de las fracciones liberadas a baja temperatura, los datos obtenidos en todos los experimentos se combinaron en el diagrama de correlación (fig. 4f) para definir una edad de isócrona de $30.6 \pm 0.4 \mathrm{Ma}$, la cual se tomó como la mejor estimación de la edad de la toba Los Azabaches. Esta nueva edad pone en evidencia el inicio de la actividad volcánica desde el Oligoceno en la UIGC que resulta más antigua que lo previamente reportado por Hausback (1984). La toba La Capilla que corona a la secuencia C-UIGC, presenta un color blanco con un espesor de 2 a $20 \mathrm{~m}$. Corresponden a tobas félsicas (riolíticas o riodacíticas) de grano fino, bien seleccionadas con una edad K/Ar en biotita en el Cerro El Indio de 22.0 \pm 0.4 Ma y otra al NW del área de estudio de $20.3 \pm 0.6$ Ma (Hausback, 1984) (tabla 2). Las edades antes mencionadas de la Toba La Capilla deben tomarse con reserva ya que seguramente son más antiguas que la toba El Oro de $22.0 \pm 0.4 \mathrm{Ma}$ (fig. 4 y tabla 3), la cual se encuentra estratigráficamente sobreyaciéndola y separada por casi $50 \mathrm{~m}$ de sedimentos.

En la localidad Punta Arranca Cabellos la toba La Capilla presenta líticos que varían de gránulos a bloques de $2 \mathrm{~cm}$ hasta más de $20 \mathrm{~m}$ (fig. 6). La composición de dichos líticos varía, algunos corresponden a bloques cognados (bloques sedimentarios arrastrados por el flujo), mientras que otros corresponden a bloques accidentales que corresponden a la estratigrafía del lugar (depósitos de la UIGC). Los bloques accidentales se encuentran deformados de manera semiplástica, en donde bloques de conglomerados y areniscas fueron integrados al flujo y deformados de manera plástica. Los rasgos texturales sugieren que los bloques cognados fueron arrastrados por el mismo flujo piroclástico, Una segunda interpretación puede considerarlos como depósitos de detritos de avalancha (Cas \& Wright, 1988), producto del colapso de un posible estratovolcán localizado fuera del área estudiada en el margen este de la península de Baja California, donde Hausback (1984) sugiere que se localizan facies volcánicas proximales y de núcleo. 
Tabla 3.-Resultados ${ }^{40} \mathrm{Ar} /{ }^{39} \mathrm{Ar}$

Feldespato RM 0105 (fusión en una etapa)

\begin{tabular}{lcccc}
\hline${ }^{40} \mathrm{Ar}^{*}{ }^{\beta 9} \mathrm{Ar}_{\mathrm{K}}$ & $\mathrm{t}$ & $\%{ }^{40} \mathrm{Ar} *$ & ${ }^{40} \mathrm{Ar} /{ }^{36} \mathrm{Ar}$ & ${ }^{37} \mathrm{Ar}_{\mathrm{Ca}} \beta{ }^{\beta 9} \mathrm{Ar}_{\mathrm{K}}$ \\
\hline $3.61 \pm 0.09$ & $22.0 \pm 0.6$ & 55.30 & 661.08 & 6.80 \\
$3.63 \pm 0.04$ & $22.1 \pm 0.3$ & 70.72 & 1009.25 & 8.19 \\
\hline
\end{tabular}

Feldespato RM 0105 (experimento de calentamiento en etapas)

\begin{tabular}{|c|c|c|c|c|c|c|c|c|c|c|}
\hline Pot & $\%{ }^{39} \mathrm{Ar}$ & ${ }^{40} \mathrm{Ar}^{*} /{ }^{\beta 9} \mathrm{Ar}_{\mathrm{K}}$ & $\mathrm{t}(\mathrm{Ma})$ & $\%^{40} \mathrm{Ar}^{*}$ & ${ }^{40} \mathrm{Ar} /{ }^{36} \mathrm{Ar}$ & ${ }^{37} \mathrm{Ar}_{\mathrm{Ca}}{ }^{\beta 9} \mathrm{Ar}_{\mathrm{K}}$ & $\mathrm{t}_{\mathrm{i}}(\mathrm{Ma})$ & $\mathrm{t}_{\mathrm{c}}(\mathrm{Ma})$ & $\left({ }^{40} \mathrm{Ar} /{ }^{36} \mathrm{Ar}\right)_{\mathrm{i}}$ & MSWD/n \\
\hline 1.00 & 0.3394 & $3.74 \pm 0.08$ & $22.7 \pm 0.5$ & 50.36 & 595.33 & 2.16 & & & & \\
\hline 6.00 & 0.6606 & $3.68 \pm 0.06$ & $22.4 \pm 0.4$ & 75.10 & 1186.53 & 14.49 & $22.5 \pm 0.3$ & $22.0 \pm 0.4$ & $302 \pm 10$ & $0.8 / 4$ \\
\hline
\end{tabular}

Feldespato RM 0205 (fusión en una etapa)

\begin{tabular}{|c|c|c|c|c|}
\hline${ }^{40} \mathrm{Ar}^{*}{ }^{\beta 9} \mathrm{Ar}_{\mathrm{K}}$ & $\mathrm{t}$ & $\%{ }^{40} \mathrm{Ar}^{*}$ & ${ }^{40} \mathrm{Ar} /{ }^{36} \mathrm{Ar}$ & ${ }^{37} \mathrm{Ar}_{\mathrm{Ca}}{ }^{\beta 9} \mathrm{Ar}_{\mathrm{K}}$ \\
\hline $5.06 \pm 0.03$ & $30.7 \pm 0.2$ & 90.97 & 3271.12 & 0.001 \\
\hline
\end{tabular}

Feldespato RM 0205 (experimentos de calentamiento en etapas)

\begin{tabular}{|c|c|c|c|c|c|c|c|c|c|c|c|c|}
\hline Pot & $\%{ }^{39} \mathrm{Ar}$ & ${ }^{40} \mathrm{Ar}^{*} /{ }^{39} \mathrm{Ar}_{\mathrm{K}}$ & $\mathrm{t}(\mathrm{Ma})$ & & $\%{ }^{40} \mathrm{Ar}^{*}$ & ${ }^{40} \mathrm{Ar} /{ }^{\beta 6} \mathrm{Ar}$ & ${ }^{37} \mathrm{Ar}_{\mathrm{Ca}}{ }^{\beta 9} \mathrm{Ar}_{\mathrm{K}}$ & $\mathrm{t}_{\mathrm{i}}(\mathrm{Ma})$ & $\mathrm{t}_{\mathrm{p}}(\mathrm{Ma})$ & $\left({ }^{39} \mathrm{Ar}\right)_{\mathrm{p}}$ & $\mathrm{t}_{\mathrm{c}}(\mathrm{Ma})$ & $\left({ }^{40} \mathrm{Ar} /{ }^{36} \mathrm{Ar}\right)_{\mathrm{i}} \mathrm{MSWD} / \mathrm{n}$ \\
\hline 2.00 & 14.51 & $4.53 \pm 0.17$ & $27.5 \pm 1.0$ & $* *$ & 49.62 & 586.49 & 0.331 & & & & & \\
\hline 6.00 & 85.49 & $5.14 \pm 0.04$ & $31.2 \pm 0.2$ & & 90.15 & 2999.32 & 0.289 & $30.7 \pm 0.3$ & & & & \\
\hline 2.00 & 10.60 & $4.69 \pm 0.19$ & $28.5 \pm 1.1$ & $* *$ & 47.46 & 562.41 & 0.276 & & & & & \\
\hline 4.00 & 17.66 & $5.03 \pm 0.06$ & $30.5 \pm 0.4$ & & 93.58 & 4600.38 & 0.272 & & & & & \\
\hline 6.00 & 71.74 & $5.15 \pm 0.03$ & $31.2 \pm 0.2$ & & 95.55 & 6639.77 & 0.350 & $30.8 \pm 0.3$ & & & & \\
\hline 1.40 & 8.82 & $4.09 \pm 0.51$ & $24.8 \pm 3.1$ & $* *$ & 13.77 & 342.68 & 10.883 & & & & & \\
\hline 2.50 & 4.60 & $4.91 \pm 0.27$ & $29.8 \pm 1.6$ & $*$ & 87.34 & 2334.24 & 18.230 & & & & & \\
\hline 3.20 & 5.60 & $4.93 \pm 0.18$ & $29.9 \pm 1.1$ & * & 90.36 & 3064.64 & 0.700 & & & & & \\
\hline 3.50 & 9.39 & $5.02 \pm 0.11$ & $30.5 \pm 0.7$ & $*$ & 93.12 & 4295.80 & 0.729 & & & & & \\
\hline 5.00 & 66.83 & $5.10 \pm 0.04$ & $30.9 \pm 0.2$ & $*$ & 92.60 & 3993.94 & 0.893 & $30.3 \pm 0.4$ & $30.8 \pm 0.3$ & 91.18 & & \\
\hline 1.20 & 11.41 & $4.29 \pm 0.29$ & $26.0 \pm 1.7$ & $* *$ & 23.43 & 385.93 & 0.267 & & & & & \\
\hline 1.30 & 8.76 & $4.87 \pm 0.08$ & $29.6 \pm 0.5$ & & 93.12 & 4292.16 & 0.630 & & & & & \\
\hline 2.40 & 7.20 & $5.07 \pm 0.11$ & $30.8 \pm 0.6$ & $*$ & 94.37 & 5246.58 & 0.877 & & & & & \\
\hline 4.10 & 8.51 & $5.00 \pm 0.07$ & $30.4 \pm 0.4$ & $*$ & 95.29 & 6273.12 & 0.669 & & & & & \\
\hline 6.00 & 52.68 & $5.09 \pm 0.02$ & $30.9 \pm 0.1$ & $*$ & 92.99 & 4217.44 & 0.685 & $30.1 \pm 0.3$ & $30.7 \pm 0.2$ & 79.83 & $30.6 \pm 0.4$ & $332 \pm 45 \quad 1.7 / 14$ \\
\hline
\end{tabular}

Pot es la potencia del láser en Watts que se utilizó para liberar el argón. t es la edad de la fracción individual, no incluye la incertidumbre en J. $t_{i}$ edad integrada; $t_{p}$ edad de meseta, calculada con las fracciones identificadas con un asterisco $\left({ }^{39} \mathrm{Ar}\right)_{\mathrm{p}}$ es el $\%$ de ${ }^{39} \mathrm{Ar}$ liberado en el segmento del espectro de edad que comprende la meseta; $t_{c}$ edad de isócrona calculada utilizando las fracciones de argón obtenidas en todos los experimentos, excepto aquellas identificadas con dos asteriscos; $\left({ }^{40} \mathrm{Ar} /{ }^{36} \mathrm{Ar}\right)_{\mathrm{i}}$ se obtiene del inverso de la intercepción con el eje de las ordenadas, de la mejor línea recta calculada con los datos en el diagrama de correlación ( $\left.{ }^{36} \mathrm{Ar} /{ }^{40} \mathrm{Ar}\right)$ versus $\left({ }^{39} \mathrm{Ar} /{ }^{40} \mathrm{Ar}\right)$, representa la composición del ${ }^{40} \mathrm{Ar} /{ }^{36} \mathrm{Ar}$ inicial. MSWD es una medida de la bondad del ajuste de la línea recta y $\mathrm{n}$ es el número de datos utilizados. J para todas las muestras es $0.003394 \pm 0.000025$. $t_{i}$, $t_{p}$ y $t_{c}$ incluyen la incertidumbre en $J$. La mejor estimación de la edad de la muestra se identifica con negrillas.

\section{Interpretación}

La base de la unidad inferior Comondú (C-UIGC) es dominada por un ambiente de abanico aluvial distal, donde existieron canales fijos y aislados de un sistema fluvial trenzado intercalados, a su vez, por depósitos eólicos y depósitos ignimbríticos.

\section{Depósitos de la parte superior de la unidad inferior Comondú (A-UIGC)}

Los depósitos de la parte superior (A-UIGC) cubren concordantemente a los depósitos de la base de la unidad inferior Comondú (C-UIGC). Las litofacies más abundantes (65\%) son las facies de are- 
niscas (tabla 1), distribuyéndose hacia el centro y sur en el área estudiada, en menor proporción destacan las facies de conglomerados $(25 \%)$, las cuales se presentan al sur y por último un $10 \%$ para las facies de depósitos de flujos de gravedad distribuyéndose en el centro del área estudiada. Las facies de areniscas consisten en litarenitas con oolitos fosfáticos de grano medio a grueso de $1 \mathrm{a} 2 \mathrm{~m}$ de longitud y un espesor de 30 a $70 \mathrm{~cm}$. Presentan estratificación cruzada y estratificación horizontal, se encuentran intercaladas por facies de limonitas masivas de color café oscuro, de $1 \mathrm{a} 5 \mathrm{~cm}$ de espesor, con grietas de desecación a pequeña escala y por facies de conglomerados (fig. 5). Las facies de conglomerados se presentan de forma masiva, polimícticos con estratificación incipiente a bien estratificados. Los estratos son de 0.5 a $8 \mathrm{~m}$ de espesor con una longitud de decenas de metros y de forma lenticular. Los clastos son del tamaño de las gravas de 3 a $7 \mathrm{~cm}$ de diámetro, de composición volcánica y metamórfica. La forma del clasto es subangular a subredondeada de pobre a moderadamente seleccionada (fig. 5). La fábrica es de clastos soportados con gradación normal. Presentan imbrincación hacia el SW $35^{\circ}$ y SE $40^{\circ}$. Estos depósitos se encuentran coronados por la toba El Oro. En el presente trabajo la toba El Oro fue datada por ${ }^{40} \mathrm{Ar} /{ }^{\beta 9} \mathrm{Ar}$. En esta muestra (RM0105) el bajo contenido de potasio no permitió realizar experimentos de calentamiento en etapas detallados. Los datos obtenidos son consistentes tanto en el espectro de edad como en el diagrama de correlación rindiendo edades estadísticamente indistinguibles. En el diagrama ${ }^{37} \mathrm{Ar}_{\mathrm{Ca}}{ }^{\beta 9} \mathrm{Ar}_{\mathrm{K}}$ puede observarse que la composición de $\mathrm{Ca} / \mathrm{K}$ de esta muestra es homogénea (fig. $4 \mathrm{~b}$ ). La mejor estimación de la edad de la toba El Oro se toma del diagrama de correlación (fig. 4c) en donde los cuatro datos claramente se alinean $(\mathrm{MSWD}=0.8)$ para rendir una edad de isócrona de $22.0 \pm 0.4 \mathrm{Ma}$ con un $\left({ }^{40} \mathrm{Ar} /{ }^{36} \mathrm{Ar}\right)_{\mathrm{i}}$ de $302 \pm 10$.

Dicha toba presenta un espesor de $4 \mathrm{~m}$, es de color vino y de forma masiva. La matriz es de ceniza vítrea compuesta por cristales de cuarzo, plagioclasas y anfíboles. Se distribuye al sur y centro en el área estudiada, en el Arroyo El oro, Arroyo Santa Victoria y Cañada La Pedrera (fig. 6).

Coronando a la toba El Oro, de manera discordante se encuentran depósitos de brechas volcánicas de $140 \mathrm{~m}$ de espesor. Estos depósitos fueron originalmente descritos por Hausback (1984) y Aranda Gómez \& Pérez Venzor (1988) que los denomina Brecha y conglomerado volcánicos
Balandra. Se distribuye en el centro y sur en el área estudiada y se estima que fue depositada en el Mioceno inferior (20.3 $\pm 0.4 \mathrm{Ma})$, con base en una datación K/Ar realizada por Hausback (1984) en un clasto de andesita, tomado 8 kilómetros al oeste del área estudiada. Estos depósitos están constituidos por clastos de 3 a $10 \mathrm{~cm}$ de diámetro de composición andesítica (Hausback, 1984) (fig. 6). Presentan variaciones texturales y estructurales que en algunos lugares predominan los bloques angulares y en otros los clastos de forma subredondeada a redondeada. Las rocas varían de masivas hasta estratificadas y desde caóticas a moderadamente seleccionadas. Tomando en cuenta sus variaciones texturales, los depósitos que se presentan de manera caótica con una matriz de ceniza y vidrio, son considerados como depósitos de flujos de bloques y cenizas de acuerdo a la clasificación de Cas \& Wright (1988), mientras que aquellos afloramientos en donde los clastos se encuentran redondeados y la matriz es de grano medio a fino, son considerados como depósitos epiclásticos.

\section{Interpretación}

Los depósitos de la parte superior de la unidad inferior Comondú (A-UIGC), corresponden a depósitos de ríos trenzados de baja sinuosidad progradados por depósitos menores de abanico aluvial (flujos de escombros).

\section{Discusión y conclusiones}

\section{Pulsos volcánicos y ambiente sedimentario}

El análisis de facies, así como dataciones K/Ar de trabajos previos y nuevas dataciones ${ }^{40} \mathrm{Ar} /{ }^{39} \mathrm{Ar}$ permitieron interpretar la evolución del ambiente sedimentario y los pulsos volcánicos de la UIGC en Punta Coyote.

De acuerdo al análisis realizado, el área estudiada formó parte de la cuenca de arco delantero al oeste del arco volcánico de la SMO durante el Oligoceno inferior al Mioceno inferior. El ambiente sedimentario de la UIGC evolucionó inicialmente como un ambiente fluvial trenzado progradando a un ambiente de abanico aluvial donde llegaron a depositarse tres unidades tobáceas: toba Los Azabaches $\left({ }^{40} \mathrm{Ar} /{ }^{39} \mathrm{Ar}\right.$ en sanidino con $30.6 \pm 0.4 \mathrm{Ma}$ ), toba La Capilla (evidencias estratigráficas sugieren una edad más antigua 


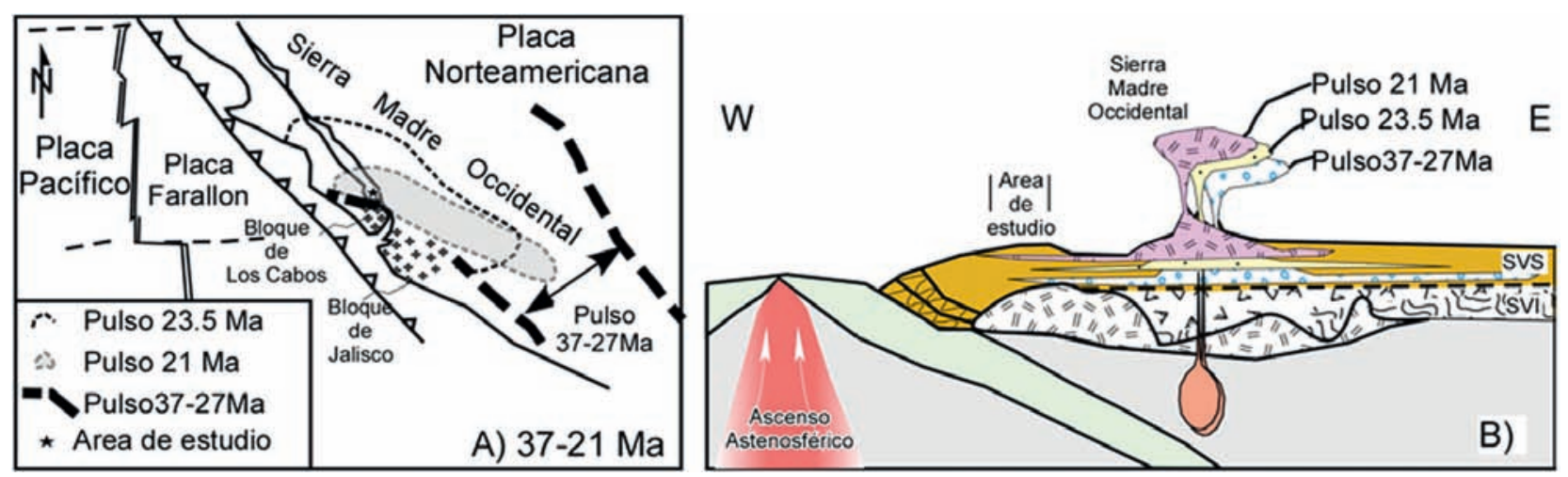

Fig. 7.-A) Reconstrucción de la geometría de placas y distribución espaciotemporal de los pulsos volcánicos de la Sierra Madre Occidental (modificado de Ferrari et al., 2002). B) Sección esquemática del margen oeste de México donde se representan los pulsos más importantes de la actividad volcánica entre los 37 y 21 Ma.

que $22.0 \pm 0.4 \mathrm{Ma})$ y la toba El Oro $\left({ }^{40} \mathrm{Ar} /{ }^{39} \mathrm{Ar}\right.$ en plagioclasa con $22.0 \pm 0.4 \mathrm{Ma}$ ) (fig. 7).

Los depósitos de la UIGC sobreyacen al Granito Sierra Las Cruces (87-94 Ma) (Frizzell et al., 1984) y están representados por depósitos que corresponden al sector distal de ambiente de abanico aluvial, donde existieron canales fijos y aislados de un sistema fluvial trenzado intercalados con depósitos arenosos y depósitos eólicos del Oligoceno superior. La composición de los clastos y la dirección de paleocorrientes de los depósitos de la parte inferior y superior de la UIGC indican la existencia de un sistema montañoso ubicado al NE del área de estudio, que corresponde a la Sierra Madre Occidental. La presencia de facies volcánicas asociadas a los canales de conglomerados y a las areniscas indica la presencia de un primer pulso volcánico de tipo ignimbrítico durante la depositación (toba Los Azabaches, $30.6 \pm 0.4 \mathrm{Ma}$ ).

Posterior al primer pulso volcánico (fig. 7) continuó la sedimentación de areniscas principalmente. Un segundo pulso volcánico originó los depósitos de la toba La Capilla (22.0 \pm 0.4 Ma, Hausback, 1984), la cual se manifiesta de manera extensa en la zona centro y norte del área de estudio. Como mencionamos en la descripción respectiva de la toba La Capilla, su edad K-Ar debe tomarse con reserva ya que considerando el error experimental puede interpretarse erróneamente que se traslapa con el pulso volcánico que generó la toba El Oro de $22.0 \pm 0.4 \mathrm{Ma}$, la cual se encuentra estratigráficamente sobreyaciéndola y separada por casi $50 \mathrm{~m}$ de sedimentos. Por lo anterior, la edad del segundo pulso volcánico es considerada como más antigua que $22.0 \pm 0.4 \mathrm{Ma}$.
Posterior al segundo pulso volcánico se produce un cambio en la fuente de abastecimiento del sedimento, indicado por la composición oolítica que presentan las areniscas de la parte superior de la UIGC. Este cambio composicional de las areniscas sugiere el levantamiento de afloramientos pertenecientes al miembro San Juan, de la Formación El Cien al nor-noreste del área de estudio. La composición de clastos de conglomerados de la parte superior de la UIGC, indica que el aporte del material se derivó de dos fuentes de abastecimiento, una situado al este y netamente volcánica (SMO) en donde los canales gravosos retrabajaron los depósitos del arco volcánico durante los períodos de mayores descargas de los ríos y otra fuente al NE (netamente mixto).

El tercer pulso volcánico (fig. 7) está representado por la toba El Oro $(22.0 \pm 0.4 \mathrm{Ma})$ que cubrió y erosionó los depósitos de la parte superior de la UIGC. Este tercer pulso revela ser muy importante a nivel local y regional. Hausback (1984) cartografió y dató una unidad ignimbrítica en la bahía de La Paz, a la cual denominó toba La Paz, con edad K/Ar $21.8 \pm 0.2$, que podría traslaparse o ser el equivalente de la toba El Oro $(22.0 \pm 0.4 \mathrm{Ma})$. A nivel regional, en el SVS de la SMO, Ferrari et al. (2002) define la secuencia del Nayar en la región de la Mesa del Nayar constituida por 11 unidades ignimbríticas con un espesor total de $\sim 1000 \mathrm{~m}$ y edades ${ }^{40} \mathrm{Ar} /{ }^{\beta 9} \mathrm{Ar}$ de 21.2 a 19.9 Ma (Ferrari et al., 2002). Dada la gran extensión geográfica de la secuencia del Nayar y las edades muy similares, así como la granulometría fina de facies distales de las tobas Los Azabaches y La Capilla, Ferrari et al. (2002) sugieren la posibilidad de que la toba La Paz tenga su origen en 
la región de la Mesa del Nayar. De acuerdo a nuestros datos la toba El Oro puede relacionarse a la toba La Paz y probablemente también tiene su origen al sureste en la Mesa del Nayar o alguna región intermedia entre la mesa del Nayar y La Paz. Según Ferrari et al. (2002) la distancia original entre La Paz y la Mesa del Nayar es difícil de estimar. Sin embargo, el borde de la caldera del Nayar queda a aproximadamente $42 \mathrm{~km}$ de la planicie costera de Nayarit, mientras que La Paz está a una distancia similar de la costa oriental de Baja California Sur. En principio, resulta posible que la toba La Paz y la toba El Oro puedan estar relacionadas a una fuente ubicada a una distancia máxima de aproximadamente $100 \mathrm{~km}$ más al este.

La toba El Oro fue cubierta por brechas volcánicas constituidas de lavas andesíticas de color ocre de edad estimada por Hausback (1984) de 20.3 $\pm 0.4 \mathrm{Ma}$.

Los pulsos volcánicos identificados en Punta Coyote pueden ser considerados como una correlación aproximada con los pulsos ignimbríticos del SVS de la SMO según Ferrari et al. (2002), con base a su edad y la posición paleogeográfica interpretada (fig. 7). Donde correlacionamos a la toba Los Azabaches $(30.6 \pm 0.4 \mathrm{Ma})$ con las rocas del arco Oligoceno (32-28 Ma) del SVS de la SMO, la toba La Capilla (22.0 $\pm 0.4 \mathrm{Ma}$, Hausback, 1984) con el pulso con pico a los $23.5 \mathrm{Ma}$ (rango 24-22 Ma) y la toba El Oro $(22.0 \pm 0.4 \mathrm{Ma})$ con el pulso con pico a los $21 \mathrm{Ma}$ (rango 17-22 Ma) del SVS de la SMO (fig. 7).

Hacia el norte del área estudiada a lo largo de la Península de Baja California, las rocas del Grupo Comondú (GC) presentan un rango de edad entre 30 a 12 Ma en la porción central (Gastil et al., 1979; McFall, 1968; McLean, 1988; Umhoefer et al., 2001), mientras que hacia el sur de la península se han reportado edades más jóvenes de 25-12 Ma (Hausback, 1984; Sawlan \& Smith, 1984; Sawlan, 1991). La UIGC en la parte central de la península y en Punta Coyote puede decirse que son comparables en edad considerando la unidad a la que Umhoefer et al. (2001) denomina unidad clástica inferior del Grupo Comondú, con una edad en su base de $29.6 \pm 3.5 \mathrm{Ma}$ (determinada con Trazas de fisión por McLean, 1988) y una edad de $22.2 \pm 0.6 \mathrm{Ma}$ ${ }^{40} \mathrm{Ar} /{ }^{39} \mathrm{Ar}$ y $20.27 \pm 0.08 \mathrm{Ma}{ }^{40} \mathrm{Ar} /{ }^{39} \mathrm{Ar}$ (Umhoefer et $a l ., 2001)$ en su parte media respectivamente.

El análisis estratigráfico de la UIGC en Punta Coyote demuestra la existencia de facies de arco delantero desde los $30.6 \pm 0.4 \mathrm{Ma}$ al sur de la Península de Baja California (Grupo Comondú). Estos nuevos datos corresponden a edades más antiguas que las reportadas previamente por Hausback, 1984. Esta nueva información complementa el conocimiento sobre la evolución estratigráfica del arco volcánico Oligoceno-Mioceno en la Península de Baja California y su correlación con los depósitos del SVS de la Sierra Madre Occidental al noroeste de México.

\section{AGRADECIMIENTOS}

Se agradece a CONACYT por el apoyo económico brindado durante el programa del postgrado del primer autor. También se agradece al Centro de Investigaciones de Ciencias Marinas (CICIMAR) el apoyo brindado durante parte del trabajo de campo.

\section{Referencias}

Aguirre Díaz, G.J. \& McDowell, F.W. (1991). The volcanic section at Nazas, Durango, Mexico, and the possibility of widespread Eocene volcanism within the Sierra Madre Occidental. Journal of Geophysical Research, 96: 13373-13388.

Allen, J.R.L. (1963). The classification of cross-stratified units, with notes on their origin. Sedimentology, 2: 93-114. doi:10.1111/j.13653091.1963.tb01204.X

Aranda Gómez, J.J. \& Pérez Venzor, J.A. (1988). Estudio geológico de Punta Coyotes, Baja California Sur. Revista del Instituto de Geología (UNAM), 7: 11-21.

Aranda Gómez, J.J.; Henry, C.D. \& Luhr, J.F. (2000). Evolución tectonomagmática post-paleocénica de la Sierra Madre Occidental y de la porción meridional de la provincial tectónica de Cuencas y Sierras. Boletín de la Sociedad Geológica Mexicana, 53: 59-71.

Atwater, T. (1970). Implications of plate tectonics for the Cenozoic tectonic evolution of western of North. Geological Society America Bulletin, 81: 3513-3536. doi:10.1130/0016-7606(1970)81[3513:IOPTFT]2.0.CO;2

Cas, R.A.F. \& Wright, J.V. (1988). Volcanic Successions: Modern and Ancient, a geological approach to processes, products and successions. Second edition. Unwin Hyman Ltd, London, 524 pp.

Cerca Martínez, L.M.; Aguirre Díaz, G. \& López Martínez, M. (2000). The geologic evolution of southern Sierra de Guanajuato, México: A documented example of the transition from the Sierra Madre Occidental to the Mexican Volcanic Belt. International Geology Review, 42: 2131-151. doi:10.1080/00206810009465073

Cowan, E.J. (1991). The large-scale architecture of the fluvial Westwater canyon member, Morrison Formation (upper Jurassic), San Juan Basin, New México. In: The Three-dimensional facies architecture of terrigenous clastic sediments and its implications for hydrocarbon discovery and recovery (Miall, A.D. \& Tyler, N., eds.). Society for Sedimentary Geology, Concepts in Sedimentology and Paleontology, 3: 80-93. 
Demant, A. (1975). Caracteres químicos principales del volcanismo Terciario y Cuaternario de Baja California Sur. Relaciones con la evolución del margen continental Pacífico de México. Revista del Instituto de Geología (UNAM), 75: 21-71.

Ferrari, L.; Valencia Moreno, M. \& Bryan, S. (2007). Magmatism and tectonics of the Sierra Madre Occidental and its relation with the evolution of the western margin of North America. In: Geology of México: Celebrating the Centenary of the Geological Society of México (Alaniz Álvarez, S.A. \& Nieto Samaniego, A.F., eds.), Geological Society of America Special Paper, 422: 1-39. doi: 10.1130/2007.2422(01)

Ferrari, L.; López Martínez, M. \& Rosas Elguera, J. (2002). Ignimbrite flareup and deformation in the southern Sierra Madre Occidental, western Mexico-implications for the late subduction history of the Farallon Plate. Tectonics, 21: 1035-1059. doi:10.1029/2001TC001302

Friend, P.F.; Slater, M.J. \& Williams, R.C. (1979). Vertical and lateral building of river sandstone bodies, Ebro Basin, Spain. Journal of the Geological Society, 136: 39-46. doi:10.1144/gsjgs.136.1.0039

Friend, P.F. (1983). Towards the field classification of alluvial architecture or sequence. In: Modern and ancient fluvial systems (Collinson, J.D. \& Lewin, J., eds.). Special Publication of the International Association of Sedimentologists, 6: 345-354.

Frizzell, Jr V.A.; Fox, L.K.; Mooser, F.C. \& Ort, K.M. (1984). Late Cretaceous granitoids, Cabo San Lucas Block, Baja California Sur, México. EOS, Transactions of the American Geophysical Union, 65: 1151.

Gastil, R.G.; Krummenacher, D. \& Minch, J. (1979). The record of Cenozoic volcanism around the Gulf of California. Geological Society of America Bulletin, 90: 839-857. doi:10.1130/00167606(1979)90<839:TROCVA > 2.0.CO;2

Hausback, B.P. (1984). Cenozoic volcanic and tectonic Evolution of Baja California Sur, México. In: Geology of the Baja California Peninsula (Frizzell, V.A., ed.), Society of Economic Paleontologists and Mineralogists, Pacific Section, 39: 219-236.

Hsü, K.J. (1978). Observations on landslides and relevance to modern interpretations. In: Rockslides and Avalanches 1: Natural Phenomena (Voight, B., ed.), Amsterdam, Elsevier, 70-93.

Jackson II, R.G. (1975). Velocity bedform texture patterns of meander bends in the lower Wabash River of Illinois and Indiana. Geological Society of America Bulletin, 86: 1511-1522.

doi:10.1130/00167606(1975)86<1511:VPOMBI>2.0.CO;2

Martin, A.; Fletcher, J.M.; López Martínez, M. \& Mendoza Borunda, R. (2000). Wanning Miocene subduction and arc volcanism in Baja California: the San Luis Gonzaga Volcanic field. Tectonophysics, 318: 27-51. doi:10.1016/S0040-1951(99)00305-4

Miall, A.D. (1978). Lithofacies types and vertical profile models of braided river deposits, a summary. In: Fluvial sedimentology (Miall, A.D., ed.). Canadian Society of Petroleum Geologists Memoir, 5: 597-604.

Miall, A.D. (1988). Reservoir heterogeneities in fluvial sandstones: lesson from outcrop studies. The Ameri- can Association of Petroleum Geologists Bulletin, 72: 682-697.

Miall, A.D. (1988). Facies architecture in clastic sedimentary basin. In: New perspectives in basin analysis (Kleinspehn, K. \& Paola, C., eds.). Springer, Berlin, 67-81.

Miall, A.D. (1990). Principles of Sedimentary Basin Analysis, Second edition, Springer-Verlag., New York, 668 pp.

McDowell, F.W. \& Clabaugh, S.E. (1979). Ignimbrites of the Sierra Madre Occidental and their relation to the tectonic history of western Mexico: Geological Society of America Special Paper, 180: 113-124.

McDowell, F.W. \& Keizer, R.P. (1977). Timing of midTertiary volcanism in the Sierra Madre Occidental between Durango City and Mazatlan, Mexico. Geological Society of America Bulletin, 88: 1479-1487. doi:10.1130/0016-7606(1977)88<1479:TOMVIT>2.0.CO;2

Miall, A.D. (1991). Hierarchies of architectural units in terrigenous clastic rocks and their relationship to sedimentation rate. In: The three-dimensional facies architecture of terrigenous clastic sediments and its implications for hydrocarbon discovery and recovery. (Miall, A.D. \& Tyler, N., eds.), Society for Sedimentary Geology, Concepts in Sedimentology and Paleontology, 3: 6-12.

Mina Uink, F. (1957). Bosquejo geológico del territorio sur de la Baja California. Boletín de la Asociación Mexicana de Geólogos Petroleros, 9: 139-270.

Miranda Avilés, R.; Beraldi Campesi, H.; Puy Alquiza, M.J. \& Carreño, A.L. (2005). Estromatolitos, tufas y travertinos de la sección El Morro: depósitos relacionados con la primera incursión marina en la Cuenca de Santa Rosalía, Baja California Sur. Revista Mexicana de Ciencias Geológicas, 22: 148-58.

Miranda Avilés, R.; Bourrouilh, R.; Nava Sánchez, E.; Puy Alquiza, M. \& Bourrouilh Le Jan, F. (2005). Analyse comparée de bassins sédimentaires transtensionnels: le bassin de Santa Rosalia (Basse Californie du Sud, Mexique) et de Mendibelza (Pyrénées, France). Estudios Geológicos, 61: 161-176. doi: 10.3989/egeol.05613-654

North American Commission on Stratigraphic Nomenclature (1983). North American stratigraphic code. American Association of Petroleum Geologists Bulletin, 67: 841-875.

North American Commission on Stratigraphic Nomenclature (NACSN). (2005). North American Stratigraphic Code. American Association of Petroleum Geologist Bulletin, 89: 1547-1591.

Reading, H.G. (1986). Sedimentary environments and facies. Blackwell Scientific Publications, $615 \mathrm{pp}$.

Shultz, A.W. (1984). Subaerial debris-flow deposition in the Upper Paleozoic Cutler Formation, Western Colorado. Journal of Sedimentary Research, 54: 759-772. doi: $10.1306 / 212 F 84 E F 2 B 2411 D 78648000102 C 1865 D$

Siebert, L. (1984). Large volcanic debris avalanches: Characteristics of source areas, deposits and associated eruptions: Journal of Volcanology and Geothermal Research, 22: 163-197. doi:10.1016/0377-0273(84)90002-7 
Sirkin, L.; Padilla Arredondo, G.; Pedrin Avilés, S.; Díaz Rivera, E. \& Gaitán Morán, J. (1984). Quaternary marine deposits, raised marine terraces, and tectonism in Baja California Sur, Mexico: A report on research in progress. In: Neotectonics and sea level variations in the Gulf of California area: a Symposium (Malpica, C.V.; Celis, G.S; Guerrero, G.J. \& Ortlieb, L., eds.). Instituto de Geología (UNAM), 319-339.

Stock, J.M. \& Hodges, K.V. (1989). Pre-Pliocene extension around the Gulf of California and the transfer of
Baja California to the Pacific Plate. Tectonics, 8: 99-115. doi:10.1029/TC008i001p00099

Tuta, Z.; Sutter, J.H.; Kesler, S.E. \& Ruiz, J. (1988). Geochronology of mercury, tin, and fluorite mineralization in northern Mexico. Economic Geology, 83: 1931-194. doi:10.2113/gsecongeo.83.8.1931

Recibido el 29 de junio de 2009 Aceptado el 23 de marzo de 2010 Publicado online el 18 de mayo de 2010 